\title{
An Improved Data Fusion Method Based on Weighted Belief Entropy considering the Negation of Basic Probability Assignment
}

\author{
Yong Chen, Yongchuan Tang ${ }^{D}$, and Yan Lei \\ School of Big Data and Software Engineering, Chongqing University, Chongqing 401331, China \\ Correspondence should be addressed to Yongchuan Tang; tangyongchuan@mail.nwpu.edu.cn
}

Received 18 July 2020; Revised 14 October 2020; Accepted 19 October 2020; Published 2 November 2020

Academic Editor: Jen-Chih Yao

Copyright (c) 2020 Yong Chen et al. This is an open access article distributed under the Creative Commons Attribution License, which permits unrestricted use, distribution, and reproduction in any medium, provided the original work is properly cited.

\begin{abstract}
Uncertainty in data fusion applications has received great attention. Due to the effectiveness and flexibility in handling uncertainty, Dempster-Shafer evidence theory is widely used in numerous fields of data fusion. However, Dempster-Shafer evidence theory cannot be used directly for conflicting sensor data fusion since counterintuitive results may be attained. In order to handle this issue, a new method for data fusion based on weighted belief entropy and the negation of basic probability assignment (BPA) is proposed. First, the negation of BPA is applied to represent the information in a novel view. Then, by measuring the uncertainty of the evidence, the weighted belief entropy is adopted to indicate the relative importance of evidence. Finally, the ultimate weight of each body of evidence is applied to adjust the mass function before fusing by the Dempster combination rule. The validity of the proposed method is demonstrated in accordance with an experiment on artificial data and an application on fault diagnosis.
\end{abstract}

\section{Introduction}

In recent years, considerable attention has been paid to multisensor data fusion technology or information acquisition and environment sensing, such as the wireless network [1], fault detection [2], condition monitoring [3], and image processing [4-6]. Due to the complexity of the targets, the data collected from a single sensor perform poorly when making decisions. Besides, multisensors may be affected by the complicated application environment so that they may make wrong decisions. Hence, multisource information modeling and fusion are important issues in many real applications [7]. Nevertheless, the uncertainty and imprecision are ineluctable for the real applications [8]. How to model and handle these kinds of imprecise and uncertain information remains an ongoing challenge $[9,10]$. To address this issue, numerous methods have been proposed for multisensor modeling and data fusion, including rough sets theory [11], belief function theory [12], Dempster-Shafer evidence theory [13], fuzzy set theory [14, 15], Z-numbers [16], and D-numbers [17-19] .

Dempster-Shafer evidence theory is an effective method in uncertain information modeling and processing, which was firstly proposed by Dempster [20] and had been developed by Shafer [21]. Dempster-Shafer evidence theory has been widely applied in considerable fields, such as decision-making [22-24], risk analysis [25, 26], evidence analysis [27], pattern recognition [28-30], fault diagnosis [31, 32], human reliability analysis [33], supplier selection [34], and failure mode and effects analysis [35-38]. Despite a number of advantages of Dempster-Shafer evidence theory, the classical Dempster combination rule cannot be directly used for conflicting sensor data fusion, especially when fusing highly conflicting data [39], which may lead to counterintuitive results [40]. Therefore, many researchers tend to preprocess the bodies of evidence to resolve the problem of fusing the highly conflicting evidence, e.g., to quantify the uncertainty before data fusion [41-43]. However, how to measure the uncertainty for practical applications in the framework of Dempster-Shafer evidence theory has to be identified [44-46].

As a well-known theory for uncertainty measure in the probabilistic framework, Shannon entropy attracts much attention in real applications [47-49]. Shannon entropy has also been introduced to many other fields, such as the network entropy $[50,51]$. Nevertheless, Shannon entropy is 
unable to be used directly among mass functions in the framework of Dempster-Shafer evidence theory because a mass function is a generalized probability assigned on the power set of the frame of discernment (FOD) [52]. To handle this issue, many methods for uncertainty measure in the Dempster-Shafer framework are claimed, including Yager's dissonance measure [53], Klir and Ramer's discord measure [54], Hohle's confusion measure [55], the weighted Hartley entropy [56], Klir and Parviz's strife measure [57], and George and Pal's conflict measure [58-60]. However, the existed methods may have limited efficiency in some situations [61]. In recent years, an uncertainty measure named Deng entropy was proposed [61]. Although Deng entropy has successfully overcome the weaknesses of the above methods [62] to some extent, Deng entropy fails to take the scale of FOD into consideration, which contributes to the loss of substantial and valuable information in information processing. By taking this into account, Tang et al. [52] introduced the weighted belief entropy to be related to the scale of FOD. But there is still some room for improvement to achieve more accurate fusing results.

Recently, Yin et al. [63] proposed a novel method to obtain the negation of the basic probability assignment (BPA), which can be used to measure the uncertainty of BPA. Based on that, this paper proposes an improved data fusion method by integrating the negation of BPA with the weighted belief entropy. The proposed method considers both the uncertainty measure of the negation of BPA and the uncertainty measure on the weight so that it can acquire more suitably weighted average evidence before applying the Dempster combination rule. Accordingly, the proposed method consists of the following steps. Firstly, Yin et al.'s model is used to obtain the negation of the BPA. After that, the relative importance of the evidence is attained by making use of the weighted belief entropy to obtain the uncertainty measure of the negation of the BPA. Moreover, the final weight for each body of evidence (BOE) is presented by the modified credibility degree of each body of evidence. Based on that, the weighted average evidence can be calculated. Then, the Dempster combination rule will be used to fuse the weighted average evidence. Furthermore, this new data fusion method is applied on fault diagnosis of a motor rotor to validate its capacity in real applications.

The rest of this paper is organized as follows: in Section 2, the preliminaries on Dempster-Shafer evidence theory, Shannon entropy, weighted belief entropy, negation of BPA, and some uncertainty measures in the Dempster-Shafer framework are briefly introduced; then, an improved data fusion method which is based on the weighted belief entropy and the negation of BPA is proposed in Section 3; Section 4 illustrates a numerical example to show the effectiveness of the proposed method; in Section 5, the proposed sensor data fusion method is adopted to an application in fault diagnosis; finally, Section 6 gives a conclusion.

\section{Preliminaries}

In this section, some preliminaries are briefly introduced, including Dempster-Shafer evidence theory $[20,21]$,
Shannon entropy [64], weighted belief entropy [52], the negation of BPA [63], and some other typical uncertainty measures in Dempster-Shafer framework [53-58].

2.1. Dempster-Shafer Evidence Theory. Let $\Omega=\left\{\Theta_{1}, \Theta_{2}, \ldots\right.$, $\left.\Theta_{i}, \ldots, \Theta_{N}\right\}$ be a finite nonempty set with $N$ mutually exclusive and exhaustive events, and $\Omega$ is called the frame of discernment (FOD). The power set of $\Omega$, denoted as $2^{\Omega}$, is composed of $2^{N}$ elements denoted as follows:

$2^{\Omega}=\left\{\varnothing,\left\{\theta_{1}\right\},\left\{\theta_{2}\right\}, \ldots,\left\{\theta_{N}\right\},\left\{\theta_{1}, \theta_{2}\right\}, \ldots,\left\{\theta_{1}, \theta_{2}, \ldots, \theta_{i}\right\}, \ldots, \Omega\right\}$.

A mass function $m$ is defined as a mapping from the power set $2 \Omega$ to the interval $[0,1]$, which satisfies the following conditions $[20,21]$ :

$$
\begin{aligned}
m(\varnothing) & =0, \\
\sum_{A \varepsilon \Omega} m(A) & =1 .
\end{aligned}
$$

If $m(A)>0$, then $A$ is called a focal element, and the mass function $m(A)$ represents how strongly the evidence supports the proposition $A$.

A body of evidence, also known as a basic probability assignment (BPA) or basic belief assignment (BBA), is represented by the focal sets and their associated mass value:

$$
(\mathfrak{R}, m)=\left\{A, m(A): A \in 2^{\Omega}, m(A)>0\right\},
$$

where $\Re$ is a subset of the power set $2^{\Omega}$ and each $A \in \Re$ has an associated nonzero mass value $m(A)$.

A BPA $m$ can also be represented by its associate belief function Bel and plausibility function $\mathrm{Pl}$, respectively, defined as follows:

$$
\operatorname{Bel}(A)=\sum_{\varnothing \neq B \subseteq A} m(B), \operatorname{Pl}(A)=\sum_{B \cap A \neq \varnothing} m(B) .
$$

In Dempster-Shafer evidence theory, two independent mass functions, denoted as $m_{1}$ and $m_{2}$, can be combined with Dempster combination rule defined as follows [20,21]:

$$
m(A)=\left(m_{1} \oplus m_{2}\right)(A)=\frac{1}{1-k} \sum_{B \cap C=A} m_{1}(B) m_{2}(C),
$$

where $k$ is a normalization constant representing the degree of conflict between $m_{1}$ and $m_{2}$ and $k$ is defined as follows $[20,21]$ :

$$
k=\sum_{B \cap C=\varnothing} m_{1}(B) m_{2}(C)
$$

2.2. Shannon Entropy. As an uncertainty measure of information volume in a system or process, Shannon entropy plays a central role in information theory. Shannon entropy indicates that the information volume of each piece of information is directly connected to its uncertain degree.

Shannon entropy, as the information entropy, is defined as follows [64]: 


$$
H=\sum_{i=1}^{N} p_{i} \log _{b} p_{i}
$$

where $N$ is the number of basic states, $p_{i}$ is the probability of state $i$, and $p_{i}$ satisfies $\sum_{1}^{N} p_{i}=1$. If the unit of information is bit, then $b=2$.

2.3. Weighted Belief Entropy. Weighted belief entropy is an improved measure of Deng entropy in the Dempster-Shafer framework. Compared with the original Deng entropy, weighted belief entropy focuses on the uncertain information represented by not only the mass function but also the scale of the FOD, which means less information loss in information processing. Weighted belief entropy, denoted as $E_{w d}$, is defined as follows [52]:

$$
E_{w d}(m)=-\sum_{A \subseteq X} \frac{|A| m(A)}{|X|} \log _{2} \frac{m(A)}{2^{|A|}-1},
$$

where $X$ is the FOD, $A$ is the focal element of the mass function, $|A|$ denotes the cardinality of the proposition $A$, and $|X|$ is the number of elements in FOD. If and only if the mass value is assigned to single elements, weighted belief entropy can be degenerated to Shannon entropy; in this case, the form of weighted belief entropy is as follows:

$$
\begin{aligned}
E_{w d}(m) & =-\sum_{A \subseteq X} \frac{|A| m(A)}{|X|} \log _{2} \frac{m(A)}{2^{|A|}-1} \\
& =-\sum_{A \subseteq X} m(A) \log _{2} m(A) .
\end{aligned}
$$
[52].

For more details about weighted belief entropy, refer to

2.4. The Negation of the BPA. The negation method provides a novel view to investigate the property of the mass functions. And the negation method can achieve the maximum entropy allocation in a manner [65]. Additionally, the negation method can highlight the rare focal elements in the BPA, which is significant to study, because the rare event may influence the system dramatically in some circumstances [66].

Recently, Yin et al. [63] proposed a novel method to obtain the negation of the basic probability assignment (BPA) for measuring the uncertainty of BPA. Unlike some other existing negation methods, Yin et al.'s method can be applied to not only the probability distributions but also the BPA. The main concepts are defined as follows.

Let us consider an event $E$ and $e_{i}$ denotes the $i$ th focal element. For each focal element $e_{i}, m\left(e_{i}\right)$ is the belief of the $i$ th focal element of the initial mass function and the negation of $m\left(e_{i}\right)$ is denoted by $\bar{m}\left(e_{i}\right)$. The general formula of the negation of the mass function can be derived as

$$
\bar{m}\left(e_{i}\right)=\frac{1-m\left(e_{i}\right)}{n-1},
$$

where $n$ is the number of focal element.
Actually, the negation of BPA makes use of the complement of each focal element to generate the negative one. Then, the belief of each focal element is normalized by making the sum of them equal to 1 . The negation method helps to assign the belief equally to each focal element, and if the mass function consists of only two focal elements, the negation method will exchange the belief of the two focal elements after the negation process. An example is given to show the procedure of the negation process.

Example 1. Assume the $\mathrm{FOD}=\{a, b, c\}$, for a mass function $m(a)=0.2, m(b)=0.7$, and $m(c)=0.1$, then

$$
\begin{aligned}
\bar{m}(a) & =\frac{1-m(a)}{n-1}=0.4 \bar{m}(b)=\frac{1-m(b)}{n-1}=0.15 \bar{m}(c) \\
& =\frac{1-m(c)}{n-1}=0.45 .
\end{aligned}
$$

For more details about weighted belief entropy, refer to [63].

2.5. Uncertainty Measures in Dempster-Shafer Framework. In this section, some other typical uncertainty measures in the framework of Dempster-Shafer evidence theory are briefly introduced. Assume that $X$ is the FOD, $A$ and $B$ are focal elements of the mass function, and $|A|$ denotes the cardinality of $A$. Then, the definitions of different uncertainty measures are shown as follows.

2.5.1. Hohle's Confusion Measure. Hohle's confusion measure, denoted as $C_{H}$, is defined as follows [55]:

$$
C_{H}(m)=-\sum_{A \subseteq X} m(A) \log _{2} \operatorname{Bel}(A) .
$$

2.5.2. Yager's Dissonance Measure. Yager's dissonance measure, denoted as $E_{Y}$, is defined as follows [53]:

$$
E_{Y}(m)=-\sum_{A \subseteq X} m(A) \log _{2} \mathrm{Pl}(A) .
$$

2.5.3. Dubois and Prade's Weighted Hartley Entropy. Dubois and Prade's weighted Hartley entropy, denoted as EDP, is defined as follows [56]:

$$
E_{\mathrm{DP}}(m)=\sum_{A \subseteq X} m(A) \log _{2}|A| .
$$

2.5.4. Klir and Ramer's Discord Measure. Klir and Ramer's discord measure, denoted as DKR, is defined as follows [54]:

$$
D_{\mathrm{KR}}(m)=-\sum_{A \subseteq X} m(A) \log _{2} \sum_{B \subseteq X} m(B) \frac{|A \cap B|}{|B|} .
$$


2.5.5. Klir and Parviz's Strife Measure. Klir and Parviz's strife measure, denoted as SKP, is defined as follows [57]:

$$
S_{\mathrm{KP}}(m)=-\sum_{A \subseteq X} m(A) \log _{2} \sum_{B \subseteq X} m(B) \frac{|A \cap B|}{|A|} .
$$

2.5.6. George and Pal's Conflict Measure. The total conflict measure proposed by George and $\mathrm{Pal}$, denoted as $\mathrm{TC}_{\mathrm{GP}}$, is defined as follows [58]:

$$
\mathrm{TC}_{\mathrm{GP}}(m)=\sum_{A \subseteq X} m(A) \sum_{B \subseteq X} m(B) 1-\left(\frac{|A \cap B|}{|A \cup B|}\right) .
$$

\section{The Proposed Method}

In this section, an improved data fusion approach is presented. The proposed method is based on the weighted belief entropy and the negation of BPA.

After uncertainty measure with the negation of BPA and the weighted belief entropy, the modified BOEs are fused with Dempster combination rule. Finally, decision-making is based on the fused results. The procedures for data fusion based on the weighted belief entropy and the negation of BPA are designed in Figure 1. follows:

The details of the six steps in Figure 1 are presented as

Step 1. Evidence from sensor report is modeled as the BOE.

In real applications, due to the diversity styles of information, the first step of information processing in the frame of Dempster-Shafer evidence theory mainly focuses on modeling uncertain information with BPAs.

Step 2. Calculate the negation of BPA.

In order to obtain more accurate fusing results, the negation of BPA is adopted to represent the information in this paper. In the proposed method, Yin et al.'s method is used to obtain the negation of BPA. For the $i$ th focal element $e_{i}$ in each BOE, the general formula of the negation of the mass function $\bar{m}\left(e_{i}\right)$ is calculated as follows:

$$
\bar{m}\left(e_{i}\right)=\frac{1-m\left(e_{i}\right)}{n-1}
$$

where $n$ is the number of focal elements.

Step 3. Uncertainty measure of the negation of BPA with weighted belief entropy.

The uncertainty of the negation of BPA modeled in the 2nd step needs to be measured with an appropriate method before further processing. In this paper, the weighted belief entropy is used to measure the uncertain degree of each BOE. For the $i$ th $\operatorname{BOE}(i=1,2$,
..., $m$ ), its corresponding uncertain degree with the weighted belief entropy $E_{W d}$ is calculated as follows:

$$
E_{W d}\left(m_{i}\right)=-\sum_{A \subseteq X} \frac{|A| \bar{m}_{i}(A)}{|X|} \log _{2} \frac{\bar{m}_{i}(A)}{2^{|A|}-1} .
$$

Step 4. Calculate the weight of each BOE.

The weight of each BOE can be calculated based on the value of weighted belief entropy. It is commonly accepted that the bigger the entropy is, the higher the uncertain degree will be [67]. For the $i$ th $\operatorname{BOE}(i=1,2$, $\ldots, m)$, its relative weight among all the available $m$ BOEs, denoted as $w_{i}$, is calculated as follows:

$$
w_{i}=\frac{E_{W d}\left(m_{i}\right)}{\sum_{i=1}^{m} E_{W d}\left(m_{i}\right)} .
$$

Step 5. Calculate the modified mass functions.

The modified mass function of each proposition is calculated for the final data fusion. For each proposition $A$ in the BOE, the modified mass function can be calculated as follows:

$$
m_{w}(A)=\sum_{i=1}^{m} w_{i} m_{i}(A)
$$

Step 6. Combination of the modified BPAs.

In the proposed method, the conflict among different evidences is transformed and measured by the negation of BPA and the weighted belief entropy, and now, data fusion can be accomplished via the Dempster combination rule. For each proposition $\mathrm{A}$ in the $\mathrm{BOE}$, the combination result can be got by calculating Dempster combination rule with $(m-1)$ times:

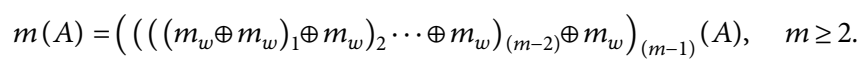

\section{Experiment with Artificial Data}

With the purpose of demonstrating the effectiveness and rationality of the proposed data fusion method, a numerical example is illustrated in this section. The experiment in [68] is recalled for the convenience of comparing with some other methods.

In target recognition, decision-making is based on reports from sensors occasionally. Considering a multisensorbased target recognition problem, three potential targets are, respectively, denoted as $A, B$, and $C$ in the FOD, denoted as $X=\{a, b, c\}$. As is shown in Table 1 , the evidence reported by five sensors is modeled as BPAs, which are denoted as $m_{1}$, $m_{2}, m_{3}, m_{4}$, and $m_{5}$. Intuitively, the report from the $2^{\text {nd }}$ sensor is contrary to the other four sensors, which may come 


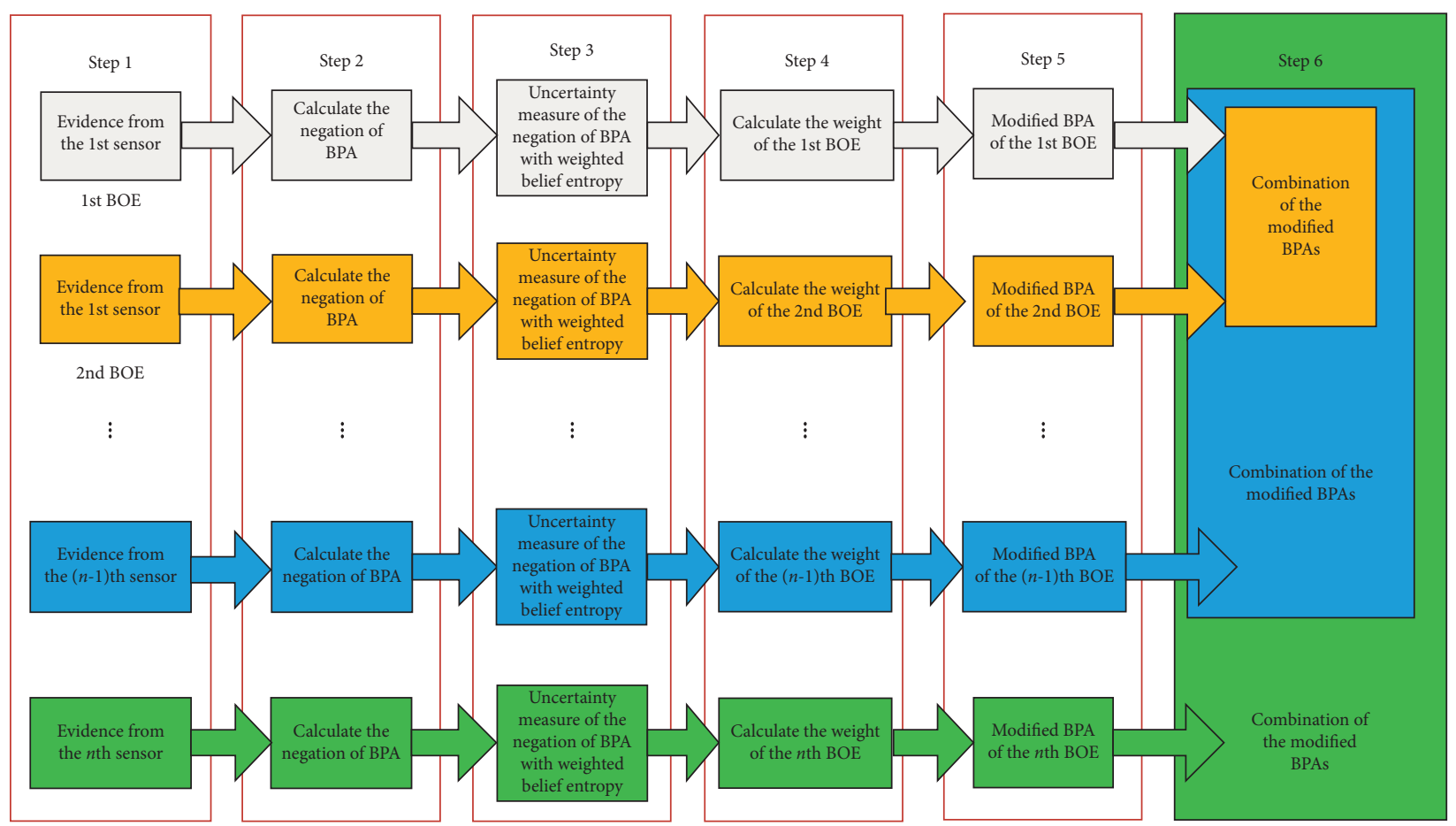

FIGURE 1: Flow chart of data fusion based on the weighted belief entropy and the negation of BPA.

TABLE 1: BPAs of artificial data.

\begin{tabular}{lcccc}
\hline BPA & $m(A)$ & $m(B)$ & $m(C)$ & $m(A, C)$ \\
\hline $1^{\text {st }}$ sensor report: $m_{1}(\cdot)$ & 0.41 & 0.29 & 0.3 & 0 \\
$2^{\text {nd }}$ sensor report: $m_{2}(\cdot)$ & 0 & 0.9 & 0.1 & 0 \\
$3^{\text {rd }}$ sensor report: $m_{3}(\cdot)$ & 0.58 & 0.07 & 0 & 0.35 \\
$4^{\text {th }}$ sensor report: $m_{4}(\cdot)$ & 0.55 & 0.1 & 0 & 0.35 \\
$5^{\text {th }}$ sensor report: $m_{5}(\cdot)$ & 0.6 & 0.1 & 0 & 0.3 \\
\hline
\end{tabular}

Execute the proposed method in Figure 1.

from a bad sensor, and $A$ will be the right target with the highest belief.

For the $1^{\text {st }}$ step, the result is adopted from [68], and the BPAs are provided in Table 1.

For the $2^{\text {nd }}$ step, with equation (18), the negation of BPA coming from the 1 st sensor is calculated as follows:

$$
\begin{aligned}
& \bar{m}(A)=\frac{1-m(A)}{n-1}=0.295, \\
& \bar{m}(B)=\frac{1-m(B)}{n-1}=0.355, \\
& \bar{m}(C)=\frac{1-m(C)}{n-1}=0.350 .
\end{aligned}
$$

Similarly, the negation of BPAs of the 2 nd sensor to the 5 th sensor can be calculated, and the results are presented in Table 2.

For the $3^{\text {rd }}$ step, the weighted belief entropy for each sensor report can be calculated with equation (8), and the calculation results are as follows:
TABLE 2: The negation of BPA.

\begin{tabular}{lcccc}
\hline BPA & $\bar{m}(A)$ & $\bar{m}(B)$ & $\bar{m}(C)$ & $\bar{m}(A, C)$ \\
\hline $1^{\text {st }}$ sensor report: $m_{1}(\cdot)$ & 0.295 & 0.2355 & 0.35 & 0 \\
$2^{\text {nd }}$ sensor report: $m_{2}(\cdot)$ & 0 & 0.1 & 0.9 & 0 \\
$3^{\text {rd }}$ sensor report: $m_{3}(\cdot)$ & 0.21 & 0.465 & 0 & 0.325 \\
$4^{\text {th }}$ sensor report: $m_{4}(\cdot)$ & 0.225 & 0.45 & 0 & 0.325 \\
$5^{\text {th }}$ sensor report: $m_{5}(\cdot)$ & 0.2 & 0.45 & 0 & 0.35 \\
\hline
\end{tabular}

$$
\begin{aligned}
& E_{W d}\left(m_{1}\right)=-\sum_{A \subseteq X} \frac{|A| \bar{m}_{1}(A)}{|X|} \log _{2} \frac{\bar{m}_{1}(A)}{2^{|A|}-1}=0.4811, \\
& E_{W d}\left(m_{2}\right)=-\sum_{A \subseteq X} \frac{|A| \bar{m}_{2}(A)}{|X|} \log _{2} \frac{\bar{m}_{2}(A)}{2^{|A|}-1}=0.2282, \\
& E_{W d}\left(m_{3}\right)=-\sum_{A \subseteq X} \frac{|A| \bar{m}_{3}(A)}{|X|} \log _{2} \frac{\bar{m}_{3}(A)}{2^{|A|}-1}=0.8546, \\
& E_{W d}\left(m_{4}\right)=-\sum_{A \subseteq X} \frac{|A| \bar{m}_{4}(A)}{|X|} \log _{2} \frac{\bar{m}_{4}(A)}{2^{|A|}-1}=0.8567 \\
& E_{W d}\left(m_{5}\right)=-\sum_{A \subseteq X} \frac{|A| \bar{m}_{5}(A)}{|X|} \log _{2} \frac{\bar{m}_{5}(A)}{2^{|A|}-1}=0.8760 .
\end{aligned}
$$

For the $4^{\text {th }}$ step, with equation (19), the weight of each $\mathrm{BOE}$ is calculated as follows: 


$$
\begin{aligned}
& w_{1}=\frac{E_{W d}\left(m_{1}\right)}{\sum_{i=1}^{5} E_{W d}\left(m_{i}\right)}=0.1459, \\
& w_{2}=\frac{E_{W d}\left(m_{2}\right)}{\sum_{i=1}^{5} E_{W d}\left(m_{i}\right)}=0.0439, \\
& w_{3}=\frac{E_{W d}\left(m_{3}\right)}{\sum_{i=1}^{5} E_{W d}\left(m_{i}\right)}=0.2712, \\
& w_{4}=\frac{E_{W d}\left(m_{4}\right)}{\sum_{i=1}^{5} E_{W d}\left(m_{i}\right)}=0.2789, \\
& w_{5}=\frac{E_{W d}\left(m_{5}\right)}{\sum_{i=1}^{5} E_{W d}\left(m_{i}\right)}=0.2593 .
\end{aligned}
$$

For the $5^{\text {th }}$ step, the weighted mass function of each proposition in Table 2 can be calculated with equation (21), and the calculation results are as follows:
TABLE 3: Experimental results with different methods.

\begin{tabular}{lcccc}
\hline Methods & $m(A)$ & $m(B)$ & $m(C)$ & $m(A, C)$ \\
\hline Deng et al.'s method [68] & 0.9820 & 0.0039 & 0.0107 & 0.0034 \\
Zhang et al.'s method [69] & 0.9820 & 0.0033 & 0.0115 & 0.0032 \\
Yuan et al.'s method [70] & 0.9886 & 0.0002 & 0.0072 & 0.0039 \\
The proposed method & 0.9890 & 0.0006 & 0.0061 & 0.0043
\end{tabular}

$$
\begin{gathered}
m_{w}(A)=\sum_{i=1}^{5} w_{i} m_{i}(A)=0.5152 \\
m_{w}(B)=\sum_{i=1}^{5} w_{i} m_{i}(B)=0.1753 \\
m_{w}(C)=\sum_{i=1}^{5} w_{i} m_{i}(C)=0.0507 \\
m_{w}(A, C)=\sum_{i=1}^{5} w_{i} m_{i}(A, C)=0.8261 .
\end{gathered}
$$

Finally, for the $6^{\text {th }}$ step, with Dempster combination rule and equation (22), each of the new weighted mass function is combined with four times. The fusion results are shown as follows:

$$
\begin{aligned}
m(A) & =\left(\left(\left(\left(m_{w} \oplus m_{w}\right)_{1} \oplus m_{w}\right)_{2} \oplus m_{w}\right)_{3} \oplus m_{w}\right)_{4}(A)=0.9890, \\
m(B) & =\left(\left(\left(\left(m_{w} \oplus m_{w}\right)_{1} \oplus m_{w}\right)_{2} \oplus m_{w}\right)_{3} \oplus m_{w}\right)_{4}(B)=0.0006, \\
m(C) & =\left(\left(\left(\left(m_{w} \oplus m_{w}\right)_{1} \oplus m_{w}\right)_{2} \oplus m_{w}\right)_{3} \oplus m_{w}\right)_{4}(C)=0.0061, \\
m(A, C) & =\left(\left(\left(\left(m_{w} \oplus m_{w}\right)_{1} \oplus m_{w}\right)_{2} \oplus m_{w}\right)_{3} \oplus m_{w}\right)_{4}(A, C)=0.0043 .
\end{aligned}
$$

The fusion results with different methods are presented in Table 3 and Figure 2.

With the proposed method, it can be inferred that target A is the right target even though the evidence $m_{2}$ has a high conflict with other evidence. With the highest belief (98.90\%), as shown in Figure 2, the proposed method works more efficiently in dealing with the conflicting evidence than the other methods in [68-70]. The reason is that the proposed method not only focuses on the scale of the FOD to reduce information loss in information processing but also considers the negation of BPA, which addresses more available uncertain information in BOE. After taking above aspects into consideration, the reliable evidence's weight is increased, while unreliable evidence's weight is decreased; therefore, its negative effect is relieved on the final fusing results than other methods.

\section{Application}

In this section, the proposed method is applied to an application on fault diagnosis for a motor rotor, where the practical data in [62] are used for the comparison with the related method.

5.1. Problem Description. According to [62], suppose the frame of discernment $\Theta$ consists of three types of fault, denoted as $F 1=\{$ rotor unbalance $\}, F 2=\{$ rotor misalignment $\}$, and $F 3=$ \{pedestal looseness\}, respectively. Three vibration acceleration sensors, which are placed in different installation positions, are used to collect the vibration signal. The acceleration vibration frequency amplitudes at the frequencies of Freq1, Freq2, and Freq 3 are taken as the fault feature variables. The collected sensor reports are provided in Table 4 , where $m_{s 1}(\cdot), m_{s 2}(\cdot)$, and $m_{s 3}(\cdot)$, respectively, denote the BOEs reported from these three vibration acceleration sensors.

5.2. Data Fusion Based on the New Method. Execute the proposed method to solve the fault diagnosis problem mentioned above:

Step 1. Evidence from sensor report is modeled as the BOE. In this paper, BPAs of sensor reports are directly adopted from [62], as is represented in Table 4. In practical applications, uncertain information modeling with BPAs has yet to be identified [71], which is not the domain of this paper. For more details about generating BPAs of Table 4, refer to [62].

Step 2. Calculate the negation of BPA.

In the proposed method, Yin et al.'s method is used to obtain the negation of BPA. With equation (18), the negation of $\mathrm{BPA}$ of each $\mathrm{BOE}$ under the vibration acceleration frequency of Freq1 is calculated as follows: 


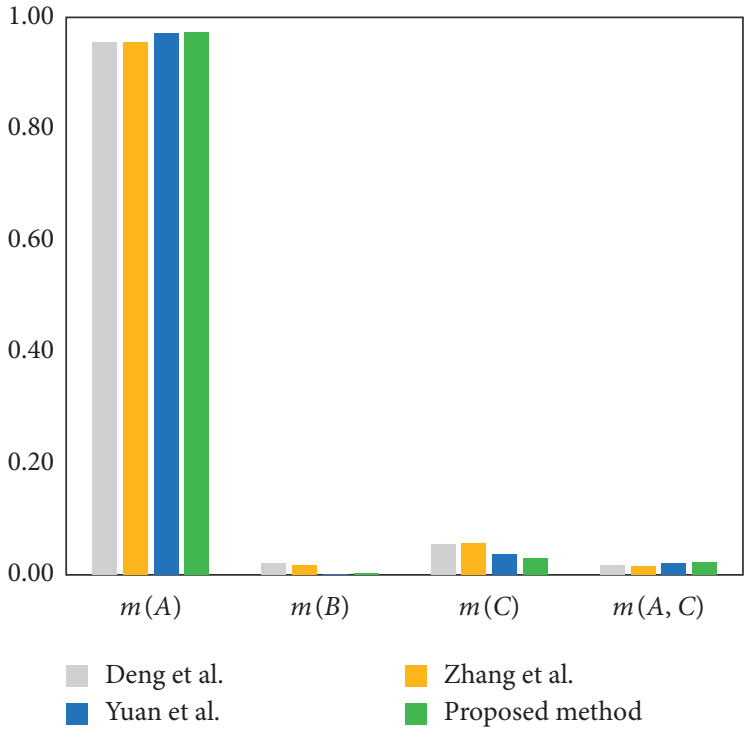

(a)

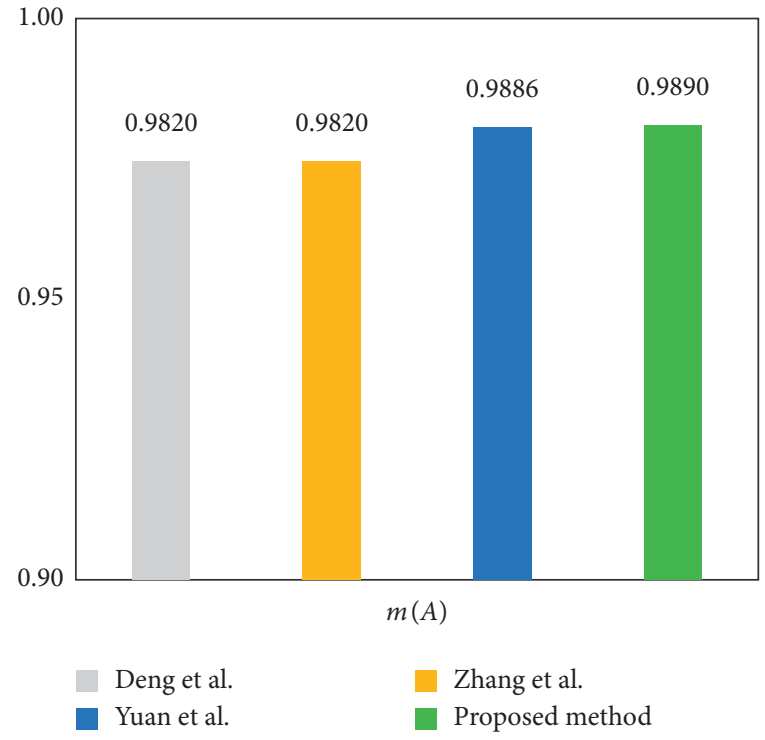

(b)

Figure 2: Comparison of fusion results generated by different methods in experiment: (a) fusion result for different objectives; (b) fusion result for target $\mathrm{A}$.

$$
\begin{aligned}
& \bar{m}_{s 1}(\{F 2\})=\frac{1-m_{s 1}(\{F 2\})}{n-1}=0.0608, \\
& \bar{m}_{s 1}(\{F 3\})=\frac{1-m_{s 1}(\{F 3\})}{n-1}=0.3332 \text {, } \\
& \bar{m}_{s 1}(\{F 1, F 2\})=\frac{1-m_{s 1}(\{F 1, F 2\})}{n-1}=0.2816 \text {, } \\
& \bar{m}_{s 1}(\{F 1, F 2, F 3\})=\frac{1-m_{s 1}(\{F 1, F 2, F 3\})}{n-1}=0.3244 \text {, } \\
& \bar{m}_{s 2}(\{F 2\})=\frac{1-m_{s 2}(\{F 2\})}{n-1}=0.1447 \text {, } \\
& \bar{m}_{s 2}(\{F 3\})=\frac{1-m_{s 2}(\{F 3\})}{n-1}=0.3330 \text {, } \\
& \bar{m}_{s 2}(\{F 1, F 2\})=\frac{1-m_{s 2}(\{F 1, F 2\})}{n-1}=0.3118 \text {, } \\
& \bar{m}_{s 2}(\{F 1, F 2, F 3\})=\frac{1-m_{s 2}(\{F 1, F 2, F 3\})}{n-1}=0.2104 \text {, } \\
& \bar{m}_{s 3}(\{F 2\})=\frac{1-m_{s 3}(\{F 2\})}{n-1}=0.2532 \text {, } \\
& \bar{m}_{s 3}(\{F 3\})=\frac{1-m_{s 3}(\{F 3\})}{n-1}=0.3332 \text {, } \\
& \bar{m}_{s 3}(\{F 1, F 2\})=\frac{1-m_{s 3}(\{F 1, F 2\})}{n-1}=0.3286 \text {, } \\
& \bar{m}_{s 3}(\{F 1, F 2, F 3\})=\frac{1-m_{s 3}(\{F 1, F 2, F 3\})}{n-1}=0.0849 \text {. }
\end{aligned}
$$

Similarly, the negation of BPA of sensor reports under Freq 2 and Freq3 can be calculated, and the results are shown in Table 5.

Step 3. Uncertainty measure of the negation of BPA with weighted belief entropy.

The uncertainty of sensor reports is measured based on the weighted belief entropy in the proposed method. With equation (19), the weighted belief entropy of each BOE under the vibration acceleration frequency of Freq1 is calculated as follows:

$$
\begin{aligned}
& E_{W d}\left(m_{s_{1}}\right)=-\sum_{A \subseteq X} \frac{|A| \bar{m}_{s_{1}}(A)}{|X|} \log _{2} \frac{\bar{m}_{s_{1}}(A)}{2^{|A|}-1}=2.3362, \\
& E_{W d}\left(m_{s_{2}}\right)=-\sum_{A \subseteq X} \frac{|A| \bar{m}_{s_{2}}(A)}{|X|} \log _{2} \frac{\bar{m}_{s_{2}}(A)}{2^{|A|}-1}=2.0535, \\
& E_{W d}\left(m_{s_{3}}\right)=-\sum_{A \subseteq X} \frac{|A| \bar{m}_{s_{3}}(A)}{|X|} \log _{2} \frac{\bar{m}_{s_{3}}(A)}{2^{|A|}-1}=1.5829 .
\end{aligned}
$$

The weighted belief entropy of sensor reports under Freq 2 and Freq 3 can be calculated, and the results are shown in Table 6.

Step 4. Calculate the weight of each BOE.

With equation (19), for the vibration acceleration frequency of Freq1, the weight of each BOE for evidence modification is calculated as follows: 
TABLE 4: Data for fault diagnosis modeled as BPAs.

\begin{tabular}{ccccccccccc}
\hline & \multicolumn{3}{c}{ Freq1 } & \multicolumn{3}{c}{ Freq2 } & \multicolumn{3}{c}{ Freq3 } \\
& $\{F 2\}$ & $\{F 3\}$ & $\{F 1, F 2\}$ & $\{F 1, F 2, F 3\}$ & $\{F 2\}$ & $\{F 1, F 2, F 3\}$ & $\{F 1\}$ & $\{F 2\}$ & $\{F 1, F 2\}$ & $\{F 1, F 2, F 3\}$ \\
\hline$m_{s 1}(\cdot)$ & 0.8176 & 0.0003 & 0.1553 & 0.0268 & 0.6229 & 0.3771 & 0.3666 & 0.4563 & 0.1185 \\
$m_{s 2}(\cdot)$ & 0.5658 & 0.0009 & 0.0646 & 0.3687 & 0.7660 & 0.2341 & 0.2793 & 0.4151 & 0.2652 \\
$m_{s 3}(\cdot)$ & 0.2403 & 0.0004 & 0.0141 & 0.7452 & 0.8598 & 0.1402 & 0.2897 & 0.4331 & 0.2470 & 0.0404 \\
\hline
\end{tabular}

TABLE 5: The negation of BPAs.

\begin{tabular}{ccccccccccc}
\hline \multicolumn{9}{c}{ Freq1 } & \multicolumn{3}{c}{ Freq2 } & \multicolumn{3}{c}{ Freq3 } \\
\hline & $\{F 2\}$ & $\{F 3\}$ & $\{F 1, F 2\}$ & $\{F 1, F 2, F 3\}$ & $\{F 2\}$ & $\{F 1, F 2, F 3\}$ & $\{F 1\}$ & $\{F 2\}$ & $\{F 1, F 2\}$ & $\{F 1, F 2, F 3\}$ \\
$m_{s 1}(\cdot)$ & 0.0608 & 0.3332 & 0.2816 & 0.3244 & 0.3771 & 0.6229 & 0.2111 & 0.1812 & 0.2938 & 0.3138 \\
$m_{s 2}(\cdot)$ & 0.1447 & 0.3330 & 0.3118 & 0.2104 & 0.2341 & 0.7659 & 0.2402 & 0.1950 & 0.2449 & 0.3199 \\
$m_{s 3}(\cdot)$ & 0.2532 & 0.3332 & 0.3286 & 0.0849 & 0.1402 & 0.8598 & 0.2367 & 0.1890 & 0.2511 & 0.3232 \\
\hline
\end{tabular}

TABLE 6: Weighted belief entropy of sensor reports under different frequencies.

\begin{tabular}{lccc}
\hline$E_{W d}(\cdot)$ & Freq1 & Freq2 & Freq3 \\
\hline$E_{W d}\left(m_{s 1}\right)$ & 2.3363 & 2.3509 & 2.3690 \\
$E_{W d}\left(m_{s 2}\right)$ & 2.0535 & 2.6083 & 2.3322 \\
$E_{W d}\left(m_{s 3}\right)$ & 1.5829 & 2.7336 & 2.3486 \\
\hline
\end{tabular}

$$
\begin{aligned}
& w_{S_{1}}=\frac{E_{W d}\left(m_{s_{1}}\right)}{\sum_{i=1}^{3} E_{W d}\left(m_{s_{i}}\right)}=\frac{2.3363}{2.3363+2.0535+1.5829}=0.3912, \\
& w_{S_{2}}=\frac{E_{W d}\left(m_{s_{2}}\right)}{\sum_{i=1}^{3} E_{W d}\left(m_{s_{i}}\right)}=\frac{2.0535}{2.3363+2.0535+1.5829}=0.3438, \\
& w_{S_{3}}=\frac{E_{W d}\left(m_{s_{3}}\right)}{\sum_{i=1}^{3} E_{W d}\left(m_{s_{i}}\right)}=\frac{1.5829}{2.3363+2.0535+1.5829}=0.2650 .
\end{aligned}
$$

The weight of different sensor reports under Freq 2 and Freq3 is shown in Table 7.

Step 5. Calculate the modified mass functions.

With equation (21), the modified mass function for each judgement on fault types with respect to Freq1 can be calculated as follows:

$$
\begin{aligned}
m_{w}(\{F 2\}) & =\sum_{i=1}^{3} w_{s i} m_{i}(\{F 2\})=0.3911 * 0.8176+0.3438 * 0.5658+0.2650 * 0.2403=0.5780 \\
m_{w}(\{F 3\}) & =\sum_{i=1}^{3} w_{s i} m_{i}(\{F 3\})=0.3911 * 0.0003+0.3438 * 0.0009+0.2650 * 0.0004=0.0005 \\
m_{w}(\{F 1, F 2\}) & =\sum_{i=1}^{3} w_{s i} m_{i}(\{F 1, F 2\})=0.3911 * 0.1553+0.3438 * 0.0646+0.2650 * 0.0141=0.0867 \\
m_{w}(\{F 1, F 2, F 3\}) & =\sum_{i=1}^{3} w_{s i} m_{i}(\{F 1, F 2, F 3\})=0.3911 * 0.0268+0.3438 * 0.3687+0.2650 * 0.7452=0.3347
\end{aligned}
$$

The modified mass function for Freq 2 and Freq 3 can also be calculated with equation (21), and the result is shown in Table 8.

Step 6. Combination of the modified BPAs.

With equation (22), for the vibration acceleration frequency of Freq1, the modified mass function will be fused with Dempster combination rule by 2 times, shown as follows:

$$
\begin{aligned}
m(\{F 2\}) & =\left(\left(m_{w} \oplus m_{w}\right)_{1} \oplus m_{w}\right)_{2}(\{F 2\})=0.9248, \\
m(\{F 3\}) & =\left(\left(m_{w} \oplus m_{w}\right)_{1} \oplus m_{w}\right)_{2}(\{F 3\})=0.0002, \\
m(\{F 1, F 2\}) & =\left(\left(m_{w} \oplus m_{w}\right)_{1} \oplus m_{w}\right)_{2}(\{F 1, F 2\})=0.0374, \\
m(\{F 1, F 2, F 3\}) & =\left(\left(m_{w} \oplus m_{w}\right)_{1} \oplus m_{w}\right)_{2}(\{F 1, F 2, F 3\})=0.0376 .
\end{aligned}
$$

The fusion results for Freq 2 and Freq 3 are shown in Table 9. 
TABLE 7: Weighted belief entropy of sensor reports under different frequencies.

\begin{tabular}{lccr}
\hline$\omega_{S i}$ & Freq1 & Freq2 & Freq1 \\
\hline$\omega_{S i}$ & 0.3911 & 0.3056 & 0.3360 \\
$\omega_{S i}$ & 0.3438 & 0.3391 & 0.3308 \\
$\omega_{S i}$ & 0.2650 & 0.3553 & 0.3331 \\
\hline
\end{tabular}

TABLE 8: Modified mass function.

\begin{tabular}{ccccccccccc}
\hline & \multicolumn{3}{c}{ Freq1 } & \multicolumn{2}{c}{ Freq2 } & \multicolumn{2}{c}{ Freq3 } \\
\hline & $\{F 2\}$ & $\{F 3\}$ & $\{F 1, F 2\}$ & $\{F 1, F 2, F 3\}$ & $\{F 2\}$ & $\{F 1, F 2, F 3\}$ & $\{F 1\}$ & $\{F 2\}$ & $\{F 1, F 2\}$ & $\{F 1, F 2, F 3\}$ \\
$m_{w}(\cdot)$ & 0.5780 & 0.0005 & 0.0870 & 0.3347 & 0.7556 & 0.2444 & 0.3121 & 0.4349 & 0.2098 & 0.0431 \\
\hline
\end{tabular}

TABLE 9: Sensor data fusion results for fault diagnosis.

\begin{tabular}{|c|c|c|c|c|c|c|c|c|c|c|}
\hline & \multicolumn{4}{|c|}{ Freq1 } & \multicolumn{2}{|c|}{ Freq2 } & \multicolumn{4}{|c|}{ Freq3 } \\
\hline & $\{F 2\}$ & $\{F 3\}$ & $\{F 1, F 2\}$ & $\{F 1, F 2, F 3\}$ & $\{F 2\}$ & $\{F 1, F 2, F 3\}$ & $\{F 1\}$ & $\{F 2\}$ & $\{F 1, F 2\}$ & $\{F 1, F 2, F 3\}$ \\
\hline Fusion result & 0.9248 & 0.0002 & 0.0374 & 0.0376 & 0.9855 & 0.0146 & 0.3353 & 0.6316 & 0.0329 & 0.0002 \\
\hline
\end{tabular}

TABLE 10: Sensor data fusion results of different methods.

\begin{tabular}{lcccccccccc}
\hline & \multicolumn{3}{c}{ Freq1 } & \multicolumn{3}{c}{ Freq2 } & \multicolumn{3}{c}{ Freq3 } \\
\hline Method & $\{F 2\}$ & $\{F 3\}$ & $\{F 1, F 2\}$ & $\{F 1, F 2, F 3\}$ & $\{F 2\}$ & $\{F 1, F 2, F 3\}$ & $\{F 1\}$ & $\{F 2\}$ & $\{F 1, F 2\}$ & $\{F 1, F 2, F 3\}$ \\
Method in [62] & 0.8861 & 0.0002 & 0.0582 & 0.0555 & 0.9621 & 0.0371 & 0.3384 & 0.5904 & 0.0651 & 0.0061 \\
Proposed method & 0.9248 & 0.0002 & 0.0374 & 0.0376 & 0.9855 & 0.0146 & 0.3353 & 0.6316 & 0.0329 & 0.0002 \\
\hline
\end{tabular}

5.3. Discussion. According to Table 9, the result of fault diagnosis is that $F 2$ is the recognized target. The conflict of sensor reports in the problem is overcome with the proposed method. For example, under Freq2, the belief on F2 is $0.8176,0.5658$, and 0.2403 , respectively, while the fusion result is 0.9248 . Moreover, the fusion result is in accordance with the method in [62] based on Table 10. Additionally, the proposed method has a higher belief degree on fault type F2 (92.48\%) than Jiang et al.'s method, as shown in Table 10.

A few reasons that contribute to the superiority and effectiveness of the new data fusion approach can be summarized as follows. First of all, the sensor data are preprocessed properly before applying the combination rules. This is very critical in combining conflicting evidence. Secondly, the new method based on the negation of BPA and the weighted belief entropy is capable to address more uncertain information in the Dempster-Shafer evidence theory framework, which contributes to a more accurate experiment result in comparison with [62]. Finally, the advantages of Dempster combination rule, such as satisfying the commutativity and associativity, guarantee the rationality of the fusion result.

\section{Conclusions}

In this paper, by considering both the negation of BPA and the effect of the uncertainty of evidence as the weight, a novel method for data fusion is proposed. The proposed method consists of three main procedures. Firstly, the negation of the BPA is obtained, which aims to measure the uncertainty of BPA in a novel view. Secondly, the weighted evidence based on weighted belief entropy is calculated for indicating the relative importance of evidence. Thirdly, based on the above two steps, the weighted evidence is obtained by computing the final weight of evidence. More importantly, the weighted evidence can be combined with the Dempster combination rule. Finally, a numerical example and an application on fault diagnosis are presented to demonstrate the superiority and effectiveness of the proposed method. Both numerical example and the application indicate that the method involving negation of BPA produces a more accurate sensor data fusion result than some existed methods by taking into consideration more uncertain information in the BOE. The proposed approach can be applied to solve data fusion problems in industrial applications.

\section{Data Availability}

All relevant data are within the paper.

\section{Conflicts of Interest}

The authors declare that there are no conflicts of interest regarding the publication of this paper.

\section{Acknowledgments}

The work was partially supported by the Chongqing Technology Innovation and Application Development Project (Grant no. cstc2019jscx-dxwtBX0012), National Key Research and Development Project of China (Grant no. 2019YFB2102602), and Fundamental Research Funds for the Central Universities (Grant no. 2020CDCGRJ037). 


\section{References}

[1] X. Yang, Q. Zhou, J. Wang, R. Zhou, and K.-C. Li, “An energyefficient dynamic decision model for wireless multi-sensor network," The Journal of Supercomputing, vol. 76, no. 3, pp. 1585-1603, 2020.

[2] H. Huijuan, M. Wang, Y. Tang, and Q. Li, "Research on data fusion of multi-sensors based on fuzzy preference relations," Neural Computing and Applications, vol. 31, no. 1, pp. 337346, 2019.

[3] T. Wang, G. Lu, and P. Yan, "Multi-sensors based condition monitoring of rotary machines: an approach of multidimensional time-series analysis," Measurement, vol. 134, pp. 326-335, 2019.

[4] H. Jin, M. Zhang, Z. Xiao, and Y. Li, "Multisensor image fusion based on contrast and directional features optimization," International Journal of Distributed Sensor Networks, vol. 14, no. 12, 2018.

[5] O. Janssens, M. Loccufier, and S. Van Hoecke, "Thermal imaging and vibration-based multisensor fault detection for rotating machinery," IEEE Transactions on Industrial Informatics, vol. 15, no. 1, pp. 434-444, 2018.

[6] R. Razavi-Far, E. Hallaji, M. Farajzadeh-Zanjani et al., "Information fusion and semi-supervised deep learning scheme for diagnosing gear faults in induction machine systems," IEEE Transactions on Industrial Electronics, vol. 66, no. 8, pp. 6331-6342, 2018.

[7] S. Majumder and D. K. Pratihar, "Multi-sensors data fusion through fuzzy clustering and predictive tools," Expert Systems with Applications, vol. 107, pp. 165-172, 2018.

[8] X.-L. Meng, L.-T. Gong, and J.-C. Yao, "A fuzzy evaluation approach with the quasi-ordered set: evaluating the efficiency of decision making units," Fuzzy Optimization and Decision Making, vol. 19, no. 3, pp. 297-310, 2020.

[9] X. Xu, D. Zhang, Y. Bai, L. Chang, and J. Li, "Evidence reasoning rule-based classifier with uncertainty quantification," Information Sciences, vol. 516, pp. 192-204, 2020.

[10] C. Fu, W. Chang, D. Xu, and S. Yang, "An evidential reasoning approach based on criterion reliability and solution reliability," Computers \& Industrial Engineering, vol. 128, pp. 401-417, 2019.

[11] B. Walczak and D. L. Massart, "Rough sets theory," Chemometrics and Intelligent Laboratory Systems, vol. 47, no. 1, pp. 1-16, 1999.

[12] C. Cheng and F. Xiao, "A new distance measure of belief function in evidence theory," IEEE Access, vol. 7, pp. 68607-68617, 2019.

[13] W. Jiang and W. Hu, "An improved soft likelihood function for Dempster-Shafer belief structures," International Journal of Intelligent Systems, vol. 33, no. 6, pp. 1264-1282, 2018.

[14] L. A. Zadeh, "Fuzzy sets," Information and Control, vol. 8, no. 3, pp. 338-353, 1965.

[15] L. Fei, H. Wang, L. Chen, and Y. Deng, "A new vector valued similarity measure for intuitionistic fuzzy sets based on owa operators," Iranian Journal of Fuzzy Systems, vol. 16, no. 3, pp. 113-126, 2019.

[16] Z. Ren, H. Liao, and Y. Liu, "Generalized z-numbers with hesitant fuzzy linguistic information and its application to medicine selection for the patients with mild symptoms of the COVID-19," Computers \& Industrial Engineering, vol. 145, Article ID 106517, 2020.

[17] H. Seiti, A. Hafezalkotob, and E. Herrera-Viedma, "A novel linguistic approach for multi-granular information fusion and decision-making using risk-based linguistic d numbers," Information Sciences, vol. 530, pp. 43-65, 2020.

[18] X. Zhou, Y. Hu, Y. Deng, F. T. S. Chan, and A. Ishizaka, “A dematel-based completion method for incomplete pairwise comparison matrix in AHP," Annals of Operations Research, vol. 271, no. 2, pp. 1045-1066, 2018.

[19] S. Xu, W. Jiang, X. Deng, and Y. Shou, “A modified physaruminspired model for the user equilibrium traffic assignment problem," Applied Mathematical Modelling, vol. 55, pp. 340-353, 2018.

[20] A. P. Dempster, "Upper and lower probabilities induced by a multivalued mapping," The Annals of Mathematical Statistics, vol. 38, no. 2, pp. 325-339, 1967.

[21] G. Shafer, A Mathematical Theory of Evidence, Princeton University Press, Princeton, NJ, USA, 1976.

[22] C. Fu, B. Hou, W. Chang, N. Feng, and S. Yang, "Comparison of evidential reasoning algorithm with linear combination in decision making," International Journal of Fuzzy Systems, vol. 22, no. 2, pp. 686-711, 2020.

[23] F. Xiao, "An improved method for combining conflicting evidences based on the similarity measure and belief function entropy," International Journal of Fuzzy Systems, vol. 20, no. 4, pp. 1256-1266, 2018.

[24] C. Fu, W. Chang, M. Xue, and S. Yang, "Multiple criteria group decision making with belief distributions and distributed preference relations," European Journal of Operational Research, vol. 273, no. 2, pp. 623-633, 2019.

[25] L. Zhang, L. Ding, X. Wu, and M. J. Skibniewski, "An improved Dempster-Shafer approach to construction safety risk perception," Knowledge-Based Systems, vol. 132, pp. 30-46, 2017.

[26] F.-J. Zhao, Z.-J. Zhou, C.-H. Hu, L.-L. Chang, Z.-G. Zhou, and G.-L. Li, "A new evidential reasoning-based method for online safety assessment of complex systems," IEEE Transactions on Systems, Man, and Cybernetics: Systems, vol. 48, no. 6, pp. 954-966, 2016.

[27] A. Sarabi-Jamab and B. N. Araabi, "How to decide when the sources of evidence are unreliable: a multi-criteria discounting approach in the Dempster-Shafer theory," Information Sciences, vol. 448-449, pp. 233-248, 2018.

[28] J. Ma, W. Liu, P. Miller, and H. Zhou, “An evidential fusion approach for gender profiling," Information Sciences, vol. 333, pp. 10-20, 2015.

[29] Z.-ga Liu, Yu Liu, D. Jean, and F. Cuzzolin, "Evidence combination based on credal belief redistribution for pattern classification," IEEE Transactions on Fuzzy Systems, vol. 28, no. 4, pp. 618-631, 2019.

[30] Z.-G. Liu, Q. Pan, D. Jean, and A. Martin, "Combination of classifiers with optimal weight based on evidential reasoning," IEEE Transactions on Fuzzy Systems, vol. 26, no. 3, pp. 1217-1230, 2017.

[31] L. Chen and Y. Deng, "A new failure mode and effects analysis model using Dempster-Shafer evidence theory and grey relational projection method," Engineering Applications of Artificial Intelligence, vol. 76, pp. 13-20, 2018.

[32] F. Xiao, "A novel evidence theory and fuzzy preference approach-based multi-sensor data fusion technique for fault diagnosis," Sensors, vol. 17, no. 11, p. 2504, 2017.

[33] X. Zheng and Y. Deng, "Dependence assessment in human reliability analysis based on evidence credibility decay model and Iowa operator," Annals of Nuclear Energy, vol. 112, pp. 673-684, 2018. 
[34] T. Liu, Y. Deng, and F. Chan, "Evidential supplier selection based on dematel and game theory," International Journal of Fuzzy Systems, vol. 20, no. 4, pp. 1321-1333, 2018.

[35] H. Zheng and Y. Tang, "A novel failure mode and effects analysis model using triangular distribution-based basic probability assignment in the evidence theory," IEEE Access, vol. 8, pp. 66813-66827, 2020.

[36] D. Wu and Y. Tang, "An improved failure mode and effects analysis method based on uncertainty measure in the evidence theory," Quality and Reliability Engineering International, vol. 36, no. 5, pp. 1786-1807, 2020.

[37] L. Chen, L. Diao, and J. Sang, "A novel weighted evidence combination rule based on improved entropy function with a diagnosis application," International Journal of Distributed Sensor Networks, vol. 15, no. 1, 2019.

[38] X. Xu, X. Weng, D. Xu, H. Xu, Y. Hu, and J. Li, "Evidence updating with static and dynamical performance analyses for industrial alarm system design," ISA Transactions, vol. 99, pp. 110-122, 2020.

[39] M. Jing and Y. Tang, "A new base basic probability assignment approach for conflict data fusion in the evidence theory," Applied Intelligence, pp. 1-13, 2020.

[40] Y. Deng, "Generalized evidence theory," Applied Intelligence, vol. 43, no. 3, pp. 530-543, 2015.

[41] D. Wu, Z. Liu, and Y. Tang, "A new classification method based on the negation of a basic probability assignment in the evidence theory," Engineering Applications of Artificial Intelligence, vol. 96, Article ID 103985, 2020.

[42] X. Zhou and Y. Tang, "A note on incomplete information modeling in the evidence theory," IEEE Access, vol. 7, pp. 166410-166414, 2019.

[43] H. Zheng and Y. Tang, "Deng entropy weighted risk priority number model for failure mode and effects analysis," Entropy, vol. 22, no. 3, p. 280, 2020.

[44] Y. Song, X. Wang, W. Wu, W. Quan, and W. Huang, "Evidence combination based on credibility and non-specificity," Pattern Analysis and Applications, vol. 21, no. 1, pp. 167-180, 2018.

[45] Y. Yang and D. Han, "A new distance-based total uncertainty measure in the theory of belief functions," Knowledge-Based Systems, vol. 94, pp. 114-123, 2016.

[46] Y. Song, X. Wang, J. Zhu, and L. Lei, "Sensor dynamic reliability evaluation based on evidence theory and intuitionistic fuzzy sets," Applied Intelligence, vol. 48, no. 11, pp. 3950-3962, 2018.

[47] J. P. G. Nascimento, F. A. P. Ferreira, V. Aguiar, I. Guedes, and R. N. Costa Filho, "Information measures of a deformed harmonic oscillator in a static electric field," Physica A: Statistical Mechanics and Its Applications, vol. 499, pp. 250257, 2018.

[48] S. Amit and L. Kaur, "Uncertainty and negationinformation theoretic applications," International Journal of Intelligent Systems, vol. 34, no. 6, pp. 1248-1260, 2019.

[49] M. Ostovare and M. R. Shahraki, "Evaluation of hotel websites using the multicriteria analysis of promethee and gaia: evidence from the five-star hotels of mashhad," Tourism Management Perspectives, vol. 30, pp. 107-116, 2019.

[50] Z. Chen, M. Dehmer, and Y. Shi, "A note on distance-based graph entropies,” Entropy, vol. 16, no. 10, pp. 5416-5427, 2014.

[51] S. Cao and M. Dehmer, "Degree-based entropies of networks revisited," Applied Mathematics and Computation, vol. 261, pp. 141-147, 2015.
[52] Y. Tang, D. Zhou, S. Xu, and Z. He, "A weighted belief entropy-based uncertainty measure for multi-sensor data fusion," Sensors, vol. 17, no. 4, p. 928, 2017.

[53] R. R. Yager, "Entropy and specificity in a mathematical theory of evidence," International Journal of General Systems, vol. 9, no. 4, pp. 249-260, 1983.

[54] G. J. Klir and A. Ramer, "Uncertainty in dempster-shafer theory: a critical re-examination," International Journal of General Systems, vol. 18, no. 2, pp. 155-166, 1991.

[55] U. Hohle, "Entropy with respect to plausibility measures," in Proceedings of the 12th IEEE International Symposium on Multiple-Valued Logic, pp. 167-169, IEEE, Piscataway, NJ, USA, 1982.

[56] D. Dubois and H. Prade, "A note on measures of specificity for fuzzy sets," International Journal of General Systems, vol. 10, no. 4, pp. 279-283, 1985.

[57] G. J. Klir and B. Parviz, "A note on the measure of discord," in Proceedings of the Eighth International Conference on Uncertainty in Artificial Intelligence, vol. 138-141, Stanford, CA, USA, July 1992.

[58] T. George and N. R. Pal, "Quantification of conflict in dempster-shafer framework: a new approach," International Journal of General Systems, vol. 24, no. 4, pp. 407-423, 1996.

[59] Y. Zhao, D. Ji, X. Yang, L. Fei, and C. Zhai, "An improved belief entropy to measure uncertainty of basic probability assignments based on deng entropy and belief interval," Entropy, vol. 21, no. 11, p. 1122, 2019.

[60] M. Qin, Y. Tang, and J. Wen, "An improved total uncertainty measure in the evidence theory and its application in decision making," Entropy, vol. 22, no. 4, p. 487, 2020.

[61] Y. Deng, "Deng entropy," Chaos, Solitons \& Fractals, vol. 91, pp. 549-553, 2016.

[62] W. Jiang, C. Xie, M. Zhuang, Y. Shou, and Y. Tang, "Sensor data fusion with z-numbers and its application in fault diagnosis," Sensors, vol. 16, no. 9, p. 1509, 2016.

[63] L. Yin, X. Deng, and Y. Deng, "The negation of a basic probability assignment," IEEE Transactions on Fuzzy Systems, vol. 27, no. 1, pp. 135-143, 2018.

[64] C. E. Shannon, "A mathematical theory of communication," ACM SIGMOBILE Mobile Computing and Communications Review, vol. 5, no. 1, pp. 3-55, 2001.

[65] R. R. Yager, "On the maximum entropy negation of a probability distribution," IEEE Transactions on Fuzzy Systems, vol. 23, no. 5, pp. 1899-1902, 2014.

[66] C. Baudrit, D. Dubois, and D. Guyonnet, "Joint propagation and exploitation of probabilistic and possibilistic information in risk assessment," IEEE Transactions on Fuzzy Systems, vol. 14, no. 5, pp. 593-608, 2006.

[67] W. Jiang, B. Wei, C. Xie, and D. Zhou, "An evidential sensor fusion method in fault diagnosis," Advances in Mechanical Engineering, vol. 8, no. 3, pp. 1-7, 2016.

[68] Y. Deng, W. K. Shi, Z. F. Zhu, and L. Qi, "Combining belief functions based on distance of evidence," Decision Support Systems, vol. 38, no. 3, pp. 489-493, 2004.

[69] Z. Zhang, T. Liu, D. Chen, and W. Zhang, "Novel algorithm for identifying and fusing conflicting data in wireless sensor networks," Sensors, vol. 14, no. 6, pp. 9562-9581, 2014.

[70] K. Yuan, F. Xiao, L. Fei, B. Kang, and Y. Deng, "Conflict management based on belief function entropy in sensor fusion," SpringerPlus, vol. 5, p. 638, 2016.

[71] X. Deng, Q. Liu, Y. Deng, and S. Mahadevan, “An improved method to construct basic probability assignment based on the confusion matrix for classification problem," Information Sciences, vol. 340-341, pp. 250-261, 2016. 